\title{
ATR-FTIR AND SPECTROSCOPIC METHODS FOR ANALYSIS OF BLACK SEED OIL FROM ALGINATE BEADS
}

\author{
HAMZEH ALKHATIBa, FARAHIDAH MOHAMED ${ }^{\mathrm{a}, \mathrm{b}}$, ABD ALMONEM DOOLAANEA ${ }^{\mathrm{a}, \mathrm{b}^{\mathrm{w}}}$
}

aAdvanced Drug Delivery Lab, Department of Pharmaceutical Technology, Kulliyyah of Pharmacy, International Islamic University Malaysia, bIKOP Sdn Bhd, Kulliyyah of Pharmacy, International Islamic University Malaysia, Jalan Sultan Ahmad Shah, 25200 Kuantan, Malaysia

Email: abdalmonemdoolaanea@yahoo.com

Received: 31 Jan 2018, Revised and Accepted: 19 Jul 2018

ABSTRACT

Objective: This study aimed to use attenuated total reflectance-fourier transform infrared spectroscopy (ATR-FTIR) for rapid quantification of black seed oil (BSO) from alginate beads and to develop simple analysis method for in vitro release study based on turbidity measurement.

Methods: Guluronic acid-rich (high-G) sodium alginate was used to encapsulate BSO with the aid of Tween 80 as a stabilizer. ATR-FTIR method was developed for quantification of encapsulation efficiency of BSO by applying Beer-Lambert law after selection of a few wave number combinations. UV-vis method based on measurement of emulsion turbidity at $600 \mathrm{~nm}$ was also developed to quantify BSO during the releasing from alginate beads in simulated intestine buffer.

Results: ATR-FTIR method exhibited linearity in the range of $25-300 \mathrm{mg}_{\mathrm{BSO}} / \mathrm{ml}_{\text {emulsion }}\left(\mathrm{mg}_{\mathrm{BSO}} / \mathrm{ml}_{\mathrm{E}}\right)$ with $\mathrm{R}^{2}=0.998, \mathrm{RSD}=8.4 \%, \mathrm{LOD}=0.28 \mathrm{mg} \mathrm{BSO}_{\mathrm{O}} / \mathrm{ml}_{\mathrm{E}}$ and LOQ $=0.87 \mathrm{mg}_{\mathrm{BSO}} / \mathrm{ml}_{\mathrm{E}}$. BSO-alginate beads was found to completely encapsulate BSO with around $100.5 \%$ efficiency. UV-vis method exhibited linearity in the range of $50-300 \mathrm{mg}_{\mathrm{BSO}} / \mathrm{ml}_{\mathrm{E}}, \mathrm{R}^{2}=0.9931, \mathrm{RSD}=1.34 \%, \mathrm{LOD}=0.89 \mathrm{mg}_{\mathrm{BSO}} / \mathrm{ml}_{\mathrm{E}}$, and LOQ=2.71 $\mathrm{mg}_{\mathrm{BSO}} / \mathrm{ml}_{\mathrm{E}}$. In addition, the method showed that total amount of BSO was released at $110 \mathrm{~min}$.

Conclusion: These methods are considered as a practical method for quantification of BSO for encapsulation efficacy and release. They will help to accelerate and improve routine characterization of encapsulated BSO in food and pharmaceutical technology.

Keywords: Black seed oil, Nigella sativa, ATR-FTIR, Encapsulation, Alginate, turbidity

(C) 2018 The Authors. Published by Innovare Academic Sciences Pvt Ltd. This is an open access article under the CC BY license (http://creativecommons.org/licenses/by/4.0/) DOI: http://dx.doi.org/10.22159/ijap.2018v10i5.25057

\section{INTRODUCTION}

Nigella sativa, or known as black seed/black cumin, is an annual plant that has been traditionally used in India, Arabian countries and the countries bordering the Mediterranean Sea for therapeutic purposes for a considerable number of ailments including asthma, hypertension, diabetes, inflammation, cough, bronchitis, headache, eczema, fever, dizziness and flu [1]. Traditionally, there is an Islamic belief that black seed is a treatment drug for everything except death [1]. In addition, the seed oil or extract is the point of interest since it has the protective and curative effects. Sahak et al. [2] have shown the effect of black seed oil (BSO) on learning and memory reinforcement. There are also few data reporting the benefits of black seed oil in children who were suffering from inflammation [3-5].

However, BSO has an undesirable taste. This requires formulation that will mask the taste such as by encapsulation into alginate beads. Alginate has become an established material and amongst the most versatile biopolymers used in a wide range of applications [6]. The most important application for alginate is the encapsulation of drugs due to the ability to cross-link with calcium ions. The cause of this fact is the interaction of calcium ions with guluronic acid that redounds to complexation mechanism [7]. While gas chromatography (GC) is one of the most common methods to analyze BSO [8], it is time-consuming leading to overall increase in the cost on top of expensive maintenance of the equipment. Furthermore, it is impossible to quantify BSO loaded in alginate beads using GC without preparatory step involving extraction of the oil. The latter incur the considerably long time and further difficulties. Attenuated total reflectance-fourier transform infrared spectroscopy (ATR-FTIR) technique can be an alternative quantification that is easy to use. In the food science field, ATR-FTIR has been widely applied for a numerous testing like the determination of solid non-fat content in raw milk [9], fatty acid contents of encapsulated fish oil microparticle supplement [10] and quantifying BSO in polymeric nanoparticles $[11,12]$.

It is known that the triglyceride is a major component in edible oils and fats, which has notable characteristic peaks exhibited at 2937 $\mathrm{cm}^{-1}$ and $2856 \mathrm{~cm}^{-1}$ in addition to other peaks in FTIR spectra [13]. Whereas characteristic peaks of alginate were reported to be at $3764.77 \mathrm{~cm}^{-1}, 2360.70 \mathrm{~cm}-1$, and $1126.35 \mathrm{~cm}^{-1}$ in FTIR spectra [14], of which there are no overlapping peaks with those of BSO, the edible oil of interest. Based on this, it seems feasible to explore the use of ATR-FTIR to quantify encapsulated oils in alginate including BSO which will be explained thereafter. The success of this application can be an impetus to a cheaper and reliable routine analysis for other similar application as well.

Due to its nature as oil, BSO forms emulsions in water in the presence of suitable surfactants. This is anticipated to occur when BSO or its formulation enters the human gastrointestinal track (GIT) or when subjected to simulated conditions such as in vitro release study. Pearce et al. [15] reported that there is a relationship between turbidity and concentration of an emulsion. As a consequence, it is possible to study the in vitro release of BSO using UV-vis spectrophotometer at $600 \mathrm{~nm}$ wavelength [16].

This method can be applied by passing scanning step for BSO and other ingredients since it relies on the total turbidity of the emulsion caused by the presence of BSO.

Therefore, this study aims to develop and validate two analysis methods: firstly, is for quantification of BSO from alginate beads using ATR-FTIR and secondly, for in vitro release study using the spectrophotometric method.

\section{MATERIALS AND METHODS}

\section{Materials}

Guluronic acid-rich (high-G) sodium alginate was obtained from Manugel DMB, ISP, USA. Tween 80 and calcium chloride were obtained from Merck Schuchardt OHG (Hohenbrunn, Germany). Ethylenediaminetetraacetic acid disodium salt dihydrate (EDTA) was obtained from Sigma-Aldrich (St. Louis, USA). Black seed oil was purchased from Blessed Seed SDN BHD (Kuantan, Malaysia). 


\section{Fabrication of BSO loaded in alginate beads}

Emulsion of BSO-alginate was prepared with the aid of Tween 80 as a stabilizer. Homogenization of emulsion was done by using ultrasonic processor (QSonica, USA; 3 cycles for 15 seconds each at $20 \mathrm{MHz}$ ). Then, the emulsion was dropped into $1 \% \mathrm{w} / \mathrm{v}$ calcium chloride bath using a syringe with needle size 22 gauge. The beads were kept in calcium chloride for $20 \mathrm{~min}$ with stirring. Fabricated beads were then filtered by a metal mesh for washing with distilled water [17].

\section{Quantification of BSO from alginate beads using ATR-FTIR method}

Freshly prepared, BSO-alginate beads were dissolved back to emulsion state by adding the same volume of a mixture of $0.5 \mathrm{M}$ EDTA: phosphate buffer $\mathrm{pH}=6.8$ ( $1: 1$ volume ratio). Then, the mixture was placed in the incubator shaker for $30 \mathrm{~min}$ at $150 \mathrm{rpm}$. A volume of $20 \mu \mathrm{l}$ of the broken beads emulsion was scanned by ATRFTIR. The spectrum of beads formulation was loaded into Essential FTIR software v3.10.016 (Operant LLC), being set with the selected method of analysis. The encapsulated amount of BSO in alginate beads was obtained directly by using the software, pre-loaded with data developed and validated earlier, as explained in the remainder of the method section. Finally, the encapsulation efficiency of the BSO into the beads was expressed as the percentage of the actual loading of BSO to the theoretical loading.

\section{ATR-FTIR measurement}

In order to identify the peaks with minimal interference by other materials, ATR-FTIR spectra of raw materials used in the fabrication of BSO-alginate beads were acquired. Briefly, Tween 80 was dissolved in $2 \% \mathrm{w} / \mathrm{v}$ of alginate solution, where the concentration of Tween 80 in alginate solution was $5 \% \mathrm{w} / \mathrm{v}$. Then a blank was prepared by mixing $1 \mathrm{ml}$ of alginate solution including Tween 80 with $1 \mathrm{ml}$ of $0.5 \mathrm{M}$ EDTA: phosphate buffer of $\mathrm{pH} 6.8$ (1:1 volume ratio) to simulate preparation of the samples. After that, ATR-FTIR spectra were recorded by using the Perkin-Elmer ATR-FTIR spectrometer (L1600401 Spectrum Two DTGS, Llantrisant, UK) in the range of $400-4000 \mathrm{~cm}^{-1}$ and $4 \mathrm{~cm}^{-1}$ resolution. The ambient air was used as background. Finally, liquid BSO, blank, and emulsion of blank-BSO were scanned at the same wavelength.

\section{Preparation of BSO-alginate standard mixtures for generation} of a calibration curve

The standard mixtures were prepared by making series of $5 \% \mathrm{w} / \mathrm{v}$ of Tween 80 in $2 \% \mathrm{w} / \mathrm{v}$ alginate: BSO with the following concentrations of BSO in the emulsion: 25, 50, 100, 200, 250 and 300 $\mathrm{mg} / \mathrm{ml}$. After that, $1 \mathrm{ml}$ of each concentration was mixed with $1 \mathrm{ml}$ of 0.5 M EDTA: phosphate buffer $(\mathrm{pH} 6.8)$ at 1:1 volume ratio. These mixtures were vortexed for $1 \mathrm{~min}$. Finally, $20 \mu \mathrm{l}$ of each concentration was placed to ATR-FTIR lens and scanned as described previously to generate a calibration curve.

\section{ATR-FTIR spectra processing and method development}

Quantification of BSO in the beads was determined by ATR-FTIR using Beer-Lambert law and the principle of constant ratio method as described by Reig et al. [18]. The computation was done by the software. The constant ratio method is dependent on a constant relationship between materials that have special absorption qualification in the infrared spectra of each other. To set the required method based on Beer-Lambert law using the software, the spectra of the standard mixtures, previously obtained, were loaded into the software system. To complete quantification method, the peaks and baselines were selected. As for the validation of data, forecasted values of the standard mixtures were plotted against the actual ones. Finally, peaks in the specific range were selected instead of peaks of a specific wavelength number. The former can avoid minor shifts in the peaks resulted from the instrument stability and sensitivity, as recommended by the software developer.

In this study, selection of absorption peaks for each component that appeared to be minimally interfered by the other materials was carried out, that led to a reduction of the interference between peak of BSO and other ingredients' peaks. This was achieved after comparing spectra of raw materials as shown in fig. 1. Selected peaks are shown in table 1 and fig. 2. Beer-Lambert law is the linear relationship between absorbance and concentration of an absorbing species. This was the basis to calculate the expected values by the software.

Repeatability test and relative standard deviation ( $\%$ RSD) was performed by repeating six ATR-FTIR scans for a standard mixture of 75 $\mathrm{mgBSO} / \mathrm{ml}$ E. The predicted quantitative response was calculated for each scan to find the \% RSD. Limit of detection (LOD) and limit of quantification (LOQ) were calculated based on the Equations 1 and 2 respectively:

$$
\begin{aligned}
\mathrm{LOD} & =3.3 \frac{\sigma}{\mathrm{S}} \ldots \ldots . \mathrm{Eqn} 1 . \\
\mathrm{LOQ} & =10 \frac{\sigma}{\mathrm{S}} \ldots \ldots . \mathrm{Eqn} 2 .
\end{aligned}
$$

Where $\sigma$ and S are the standard deviation of the response and the slope respectively.

To choose the best calibration curve to be applied for quantification of BSO from alginate beads, the previous RSD and $\mathrm{R}^{2}$ for each selected peaks were compared (table 1). The best curve is characterized by the $\mathrm{R}^{2}$ closest to 1 and the lowest RSD.

Development of spectroscopic method based on turbidity measurement for the release study of BSO

To generate the calibration curve, stock dilution of BSO-alginate emulsion: phosphate buffer $\mathrm{pH} 6.8$ was carried out by making 300 $\mathrm{mg} / \mathrm{ml}$ of $\mathrm{BSO} /$ emulsion then $1 \mathrm{ml}$ of the emulsion was diluted to $100 \mathrm{ml}$ by phosphate buffer. Then the dilution was placed in conical flasks and kept in the incubator shaker for $2 \mathrm{~h}$ to simulate procedure of beads breaking in phosphate buffer medium (The beads will become diluted emulsion) where also the temperature and rpm were set at $37{ }^{\circ} \mathrm{C}$ and $100 \mathrm{rpm}$ respectively. The emulsion was prepared exactly as preparation of the emulsion that was used to fabricate alginate beads to make the concentration of BSO is the only factor can influence on turbidity. Thereafter, serial dilutions were executed from the last stock dilution in the range $50-300 \mathrm{mg} / \mathrm{ml}$. After that, $1 \mathrm{ml}$ of each dilution was diluted 5 times to get absorbance less than 2 and measured by UV-vis spectrophotometer (UV-1800 240V, Shimadzu, Tokyo, Japan) at wavelength $600 \mathrm{~nm}$ using silica quartz square cell cuvette with $1 \mathrm{~cm}$ bath length (Optima, Japan). The calibration curve was constructed and $\mathrm{R}^{2}$ was recorded. Specificity was confirmed by measuring the placebo six times at $600 \mathrm{~nm}$. The placebo was made by dilution $1 \mathrm{ml}$ of $2 \% \mathrm{w} / \mathrm{v}$ alginate solution contains Tween 80 as $5 \% \mathrm{w} / \mathrm{v}$ to $100 \mathrm{ml}$ by phosphate buffer, then was treated as explained previously in terms of shaking time, temperature, and rpm. In addition, repeatability was performed by measuring the $136.07 \mathrm{mg} / \mathrm{ml}$ emulsion six times. The relative standard deviation (\%RSD) was calculated. Moreover, accuracy was calculated by measuring two known concentrations of the emulsion at $600 \mathrm{~nm}$. The recovery was calculated as a percentage of the actual concentrations. The limit of detection (LOD) and limit of quantification (LOQ) were obtained using Eqn. 1 and Eqn. 2 respectively.

\section{The in vitro release study of BSO from alginate beads}

The in vitro release study was performed in the medium of phosphate buffer pH6.8. Amount of alginate beads containing BSO, which prepared from $1 \mathrm{ml}$ emulsion was placed in a conical flask containing $99 \mathrm{ml}$ of the release medium, to make a total volume of $100 \mathrm{ml}$. The sample was incubated at $37 \pm 0.5^{\circ} \mathrm{C}$ under shaking at 100 rpm. After predetermined time points $(20,35,50,65,80,95,110,125$, 140 and $155 \mathrm{~min}), 1 \mathrm{ml}$ was collected from release medium and replaced with fresh phosphate buffer then diluted to $5 \mathrm{ml}$ by the same medium. The concentration of BSO in the solution was measured by using UV-vis at $600 \mathrm{~nm}$ [19].

\section{RESULTS AND DISCUSSION}

\section{ATR-FTIR spectra}

ATR-FTIR spectra of the raw materials are shown in fig. 1 . The notable characteristic peaks for BSO were at $2854 \mathrm{~cm}^{-1}$ (C-H in-CH2), $2923 \mathrm{~cm}^{-1}$ (C-H in-CH2), and $3009 \mathrm{~cm}^{-1}$ (C-H in $\left.\mathrm{HC}=\mathrm{CH}\right)[10,20]$ due to the dominance of carbon chains in the fatty acids. In general, more 
than $98 \%$ of BSO is fatty acids [11]. Blank did not exhibit any important absorption peak in the same exhibition range of BSO.
When BSO was mixed with blank, they exhibited the same absorption peaks of BSO.

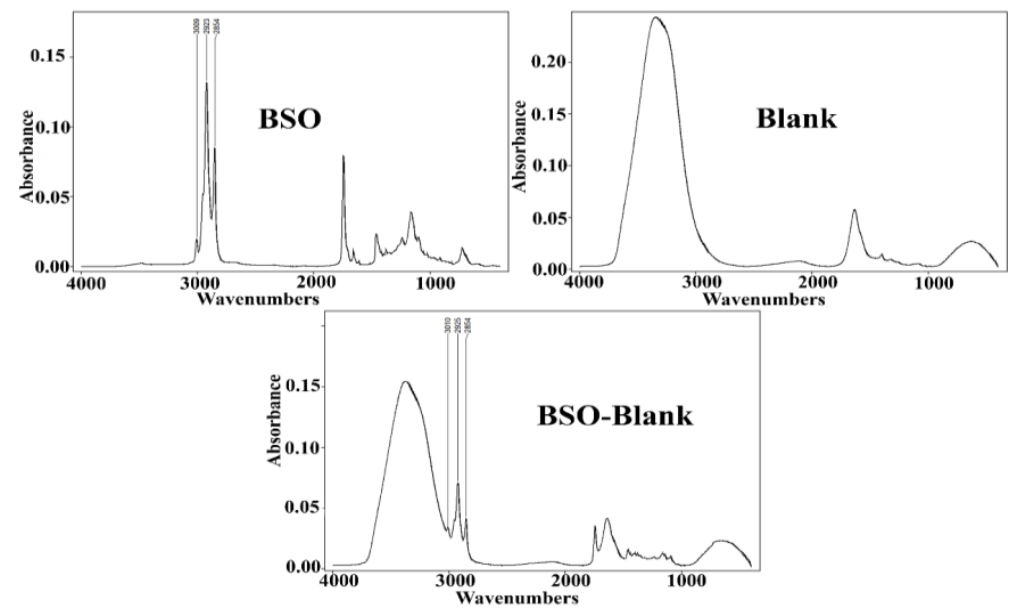

Fig. 1: ATR-FTIR Spectra of the BSO, blank, and BSO-blank, which used in BSO-alginate beads fabrication

\section{Standard curve construction}

Quantification of BSO was based on Beer-Lambert law and constant ratio method [18]. Comparing the spectra of the raw materials was carried out (fig. 1), then absorbance peaks were selected with minimal interference from the other compounds. Consequently, peak selection was made mainly by comparing the ATR-FTIR spectra of the blank, BSO and their mixture as shown in (fig. 1 and fig. 2). Table 1 shows a selection of featured peaks for BSO and the blank. To get the best standard curve, different mathematical combinations of the peaks' heights and the baseline regions were tested (table 1). Based on table 1, the best standard curve was obtained at the absorbance $2854 \mathrm{~cm}^{-1}$ with the baseline correction at $2561.029 \mathrm{~cm}^{-1}$ for BSO, and the absorbance $521.88 \mathrm{~cm}^{-1}$ with baseline correction at $802.65 \mathrm{~cm}^{-1}$ for the blank (fig. 3A). At these wavelengths, the $\mathrm{R}^{2}$ and the RSD values are the closest to $1(0.99)$ and the lowest (8.4\%) respectively. The best absorbance peak in this study $(2854 \mathrm{~cm}-1)$ was different from a previous study that found best peak for BSO quantification at $2924 \mathrm{~cm}^{-1}$ [11]. The difference was attributed to the difference in sample conditions. In this study, the sample contained a buffer in a liquid state where there was the large shoulder of the buffer peak at 3000-4000 $\mathrm{cm}^{-1}$. This peak interfered significantly with BSO peak at $\sim 2924 \mathrm{~cm}^{-1}$ but to less extent with the peak at $2854 \mathrm{~cm}^{-1}$. A previous study for quantification of fish oil using ATRFTIR utilized peaks in the region of $2800-3100 \mathrm{~cm}^{-1}$ due to the predominance of peaks related to lipid components [10].

Table 1: Selected peaks from ATR-FTIR spectra of the BSO-blank standard mixtures in order to construct the best standard curve LOD and LOQ are represented by $\% \mathrm{w} / \mathrm{w}$ BSO/blank

\begin{tabular}{|c|c|c|c|c|c|c|c|c|}
\hline BSO peak $\left(\mathrm{cm}^{-1}\right)$ & & Blank peak $\left(\mathrm{cm}^{-1}\right)$ & & $\mathbf{R}^{2}$ & RSD & LOD & LOQ & Fig. \\
\hline Peak (region type: point) & Baseline & Peak (region type: point) & Baseline & & & & & \\
\hline 2854 & 2561.029 & 521.88 & 802.65 & 0.998 & $8.40 \%$ & 0.28 & 0.87 & fig. $3 \mathrm{~A}$ \\
\hline 2854 & 2561.029 & 545.286 & 779.28 & 0.9964 & $10.04 \%$ & 0.30 & 1.006 & fig. 3B \\
\hline 2854 & 2561.029 & 562 & 779.28 & 0.994 & $13.92 \%$ & 0.53 & 1.63 & fig. 3C \\
\hline 2925 & 2561.029 & 782.629 & 1110.229 & 0.997 & $15.49 \%$ & 0.67 & 2.05 & fig. 3D \\
\hline 2925 & 2561.029 & 1635.057 & 1521.4 & 0.9892 & $13.57 \%$ & 0.65 & 1.99 & fig. $3 E$ \\
\hline
\end{tabular}

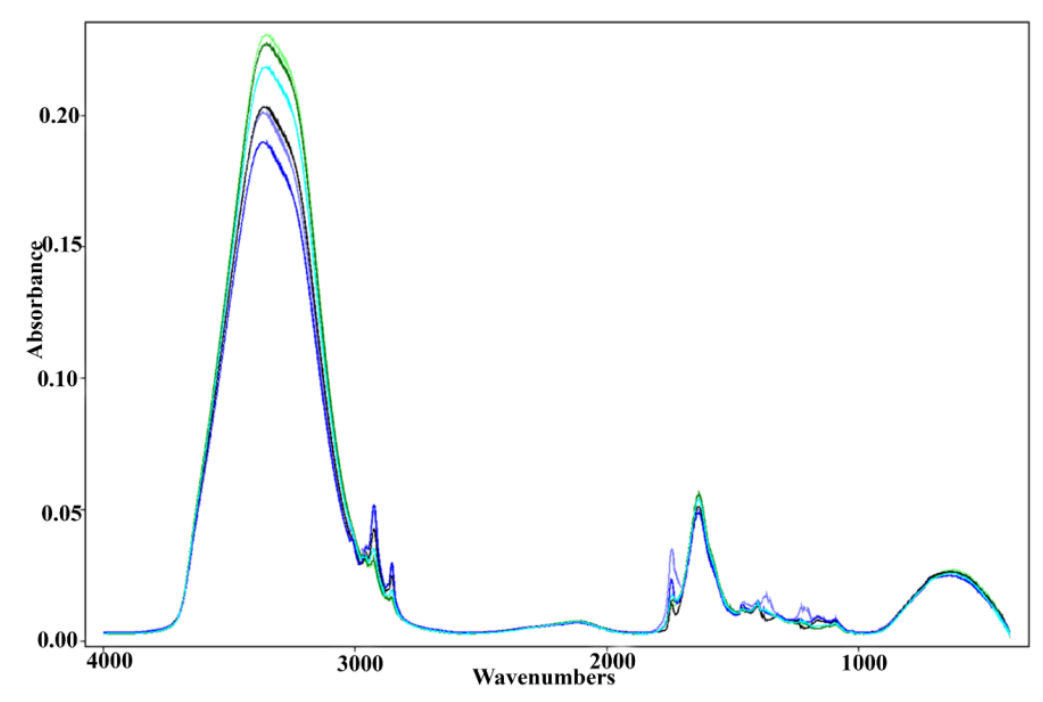

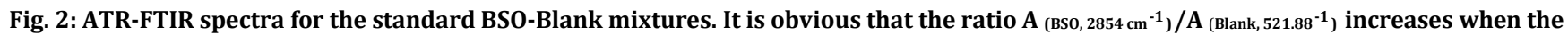
ratio BSO/Blank increases 


\section{Quantification of BSO in alginate beads}

ATR-FTIR spectra of BSO-alginate beads (5\% w/v of Tween 80 in 1.5 of BSO: 8.5 of alginate volume ratio), which become emulsion after disintegrating by using a mixture of EDTA: phosphate buffer as mention above (fig. 4), were used to quantify the loading efficiency of BSO. Based on the above mentioned best standard curve (fig. 3A), BSO loading efficiency was estimated to be $100.5 \pm 18 \%$ where the results were taken in triplicate. This result is comparable to the previous study where BSO was encapsulated in poly (L-lactic coglycolic acid) (PLGA) polymer at $101.5 \%$ to $107.0 \%[11,12]$. The loading efficiency of $100.5 \%$ seems to be acceptable based on the stability of the emulsion before fabrication of BSO-alginate beads. As for value of $\pm S D$, it can be related to the irregularity of BSO disparity in the emulsion of the dissolved beads. Maybe using emulsifiers can lead to homogeneous samples, which are subjected for scanning.
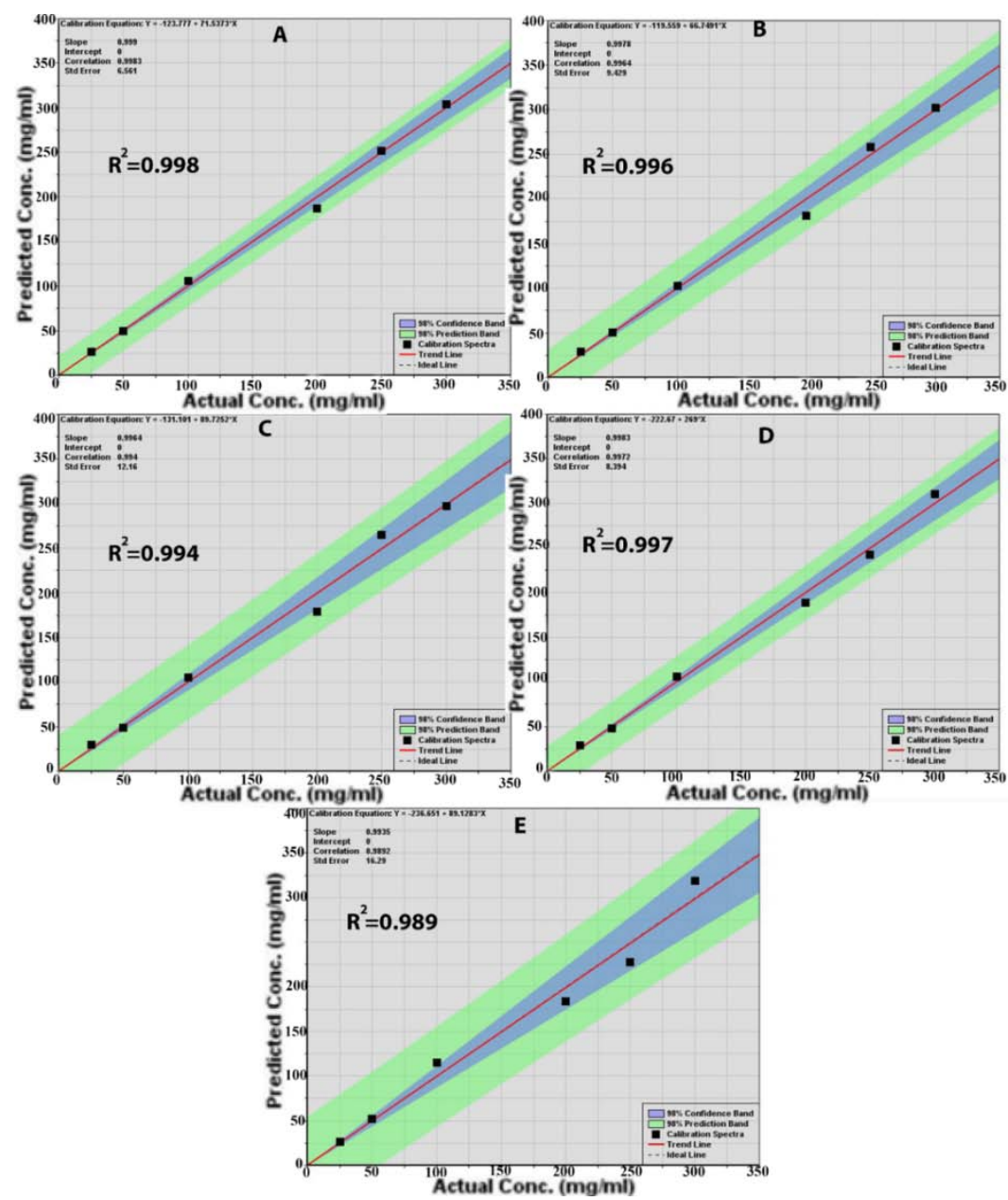

Fig. 3: Standard curves obtained from ATR-FTIR Spectra based on Beer-Lambert law. Each curve represents a set of selected peaks explained in table 1

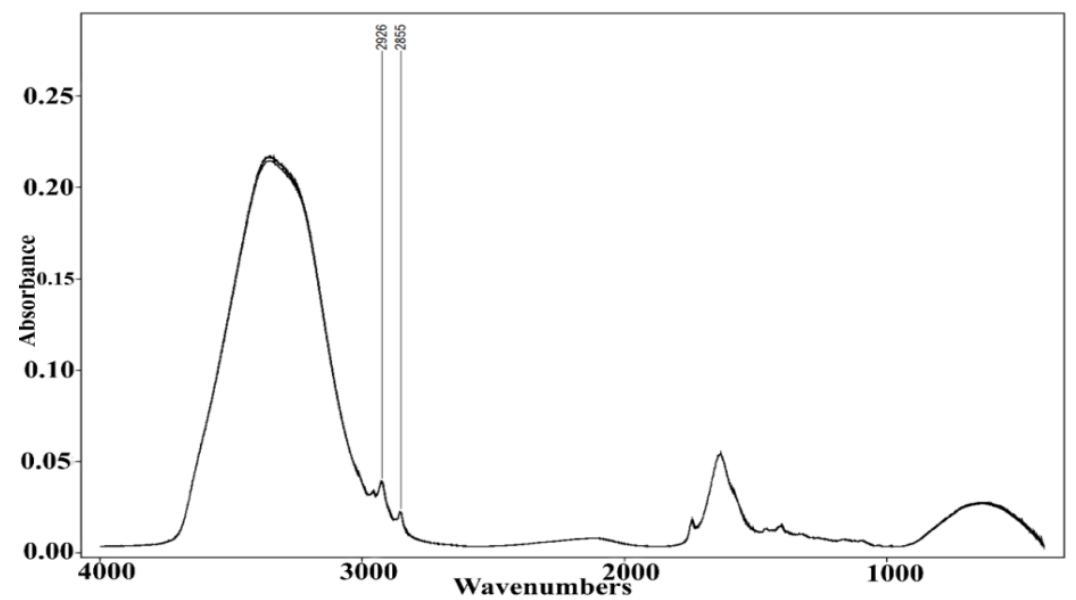

Fig. 4: ATR-FTIR spectra of BSO-alginate beads, which become emulsion after dissolving by using mixture of EDTA: phosphate buffer $(n=3)$ 


\section{Analysis method for the in vitro release of BSO form alginate beads}

The standard curve (fig. 5) was constructed using 7 different concentrations of BSO-alginate emulsion at a wavelength of $600 \mathrm{~nm}$. The response obtained from measuring the turbidity of the emulsions was found to be in the linear form in the range 50-300 $\mathrm{mg} / \mathrm{ml}$ and the equation obtained was denoted as $y=0.0038 x$ 0.0649 with the value of $\mathrm{R}^{2}$ of 0.9931 . The readings for 7 different known concentrations were taken in triplicate for each concentration.
The average of placebo absorbance was 0.002, confirming the specificity of the method. In addition, repeatability was confirmed with \%RSD of 1.34. Recovery of the two spiked concentrations (136.07 $\mathrm{mg} / \mathrm{ml}$ and $150 \mathrm{mg} / \mathrm{ml}$ ) was found to be $98.66 \%$ and $106.00 \%$, respectively. LOD and LOQ were 0.89 and $2.71 \mathrm{mg} / \mathrm{ml}$ respectively. The results suggest suitability of the turbidity method for the quantification of the release of BSO from alginate beads. This finding complies with prevthe ious study that utilized turbidity measurement to quantify the release of oil in water emulsion from alginate beads [21].

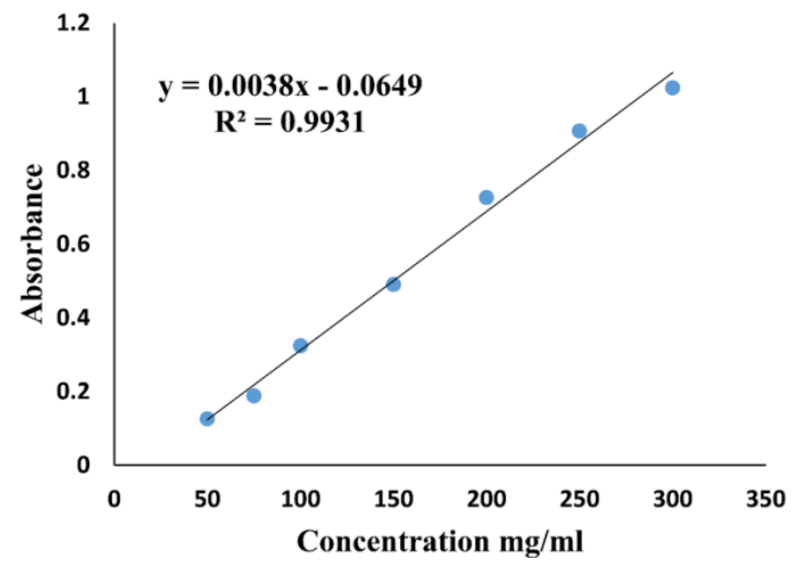

Fig. 5: The standard curve of BSO emulsion dilution using spectrophotometry

In vitro release study and encapsulation efficiency of BSO in alginate beads

Triplicate samples from the medium of release for each timing point were subjected to spectrophotometry measurement at $600 \mathrm{~nm}$. Fig. 6 shows the amount of BSO released from alginate beads during 155 min. Amount of BSO release from alginate beads for each time point was significantly increasing $(\mathrm{p}<0.05)$ based on one way ANOVA followed by Tukey test. The amount of BSO released became near to plateau at $110 \mathrm{~min}$. According to the theoretical amount of encapsulated BSO in the alginate beads, the encapsulation efficiency of BSO was $100.09 \%$. Thus, 110 min seem to be sufficient to release total amount of BSO from the fabricated beads. The relatively fast release of the oil can be attributed to the non-ionic emulsifier (Tween 80), which has weak interaction with the anionic alginate network [21].

Therefore, the beads were expected to release completely the encapsulated BSO after oral administration. This method confirmed the quantification of BSO from alginate beads by using ATR-FTIR, where both of them gave very close results.

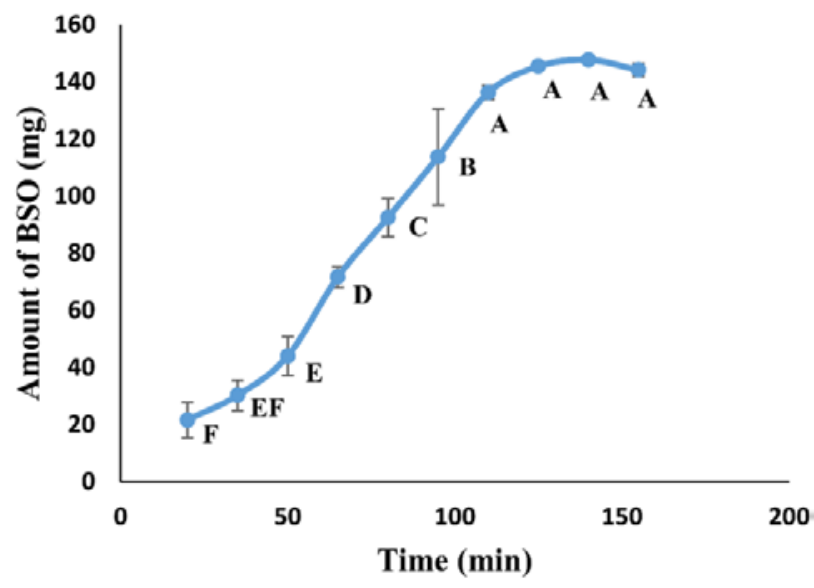

Fig. 6: Values of BSO releasing at each timing point, and grouping information based on Tukey post-hoc test. Groups with different letters are statistically significant $(p<0.05)$, values are mean $\pm S D(n=3)$

\section{ACKNOWLEDGMENT}

This work was funded by IIUM Research Initiative Grant Scheme (Gran ID: RIGS15-092-0092 and RIGS 16-114-0278).

\section{CONCLUSION}

Quantification method of BSO in alginate beads by applying ATRFTIR was found to be suitable for calculation of the encapsulation efficacy of BSO in alginate beads. Measurement of emulsion turbidity using UV-vis at $600 \mathrm{~nm}$ was found to be a feasible method to study the release of BSO from alginate beads.

The two method helps to characterize BSO in alginate beads, which can be applied in the strict routine quality control for any food and pharmaceutical company embracing Good Manufacturing Practice. 


\section{AUTHORS CONTRIBUTIONS}

All the author have contributed equally

\section{CONFLICT OF INTERESTS}

The authors declare no conflict of interest

\section{REFERENCES}

1. Ali B, G Blunden. Pharmacological and toxicological properties of Nigella sativa. Phytother Res 2003;17:299-305.

2. Sahak MKA, N Kabir, G Abbas, S Draman, NH Hashim, DS Hasan Adli. The role of Nigella sativa and its active constituents in learning and memory. J Evidence Based Complementary Altern Med 2016. http://dx.doi.org/10.1155/2016/6075679.

3. Gholamnezhad Z, R Keyhanmanesh, MH Boskabady. Antiinflammatory, antioxidant, and immunomodulatory aspects of Nigella sativa for its preventive and bronchodilatory effects on obstructive respiratory diseases: a review of the basic and clinical evidence. J Funct Foods 2015;17:910-27.

4. Srinivasan K, Cumin (Cuminum cyminum) and black cumin (Nigella sativa) seeds: traditional uses, chemical constituents, and nutraceutical effects. FQS 2018;2:1-16.

5. Tavakkoli A, V Mahdian, BM Razavi, H Hosseinzadeh. Review on clinical trials of black seed (Nigella sativa) and its active constituent, thymoquinone. J Pharmacopuncture 2017;20:107-11.

6. Tønnesen $\mathrm{HH}, \mathrm{J}$ Karlsen. Alginate in drug delivery systems. Drug Dev Ind Pharm 2002;28:621-30.

7. Suganya V, S Asheeba. Microencapsulation of astaxanthin using ionotropic gelation method isolated from three crab varieties. Int J Curr Pharm Res 2015;7:96-9.

8. Hadi MY, GJ Mohammed, IH Hameed. Analysis of bioactive chemical compounds of Nigella sativa using gas chromatographymass spectrometry. J Pharm Pharmacol 2016;8:8-24.

9. Bassbasi M, S Platikanov, R Tauler, A Oussama. FTIR-ATR determination of solid non fat (SNF) in raw milk using PLS and SVM chemometric methods. Food Chem 2014;146:250-4.

10. Vongsvivut J, P Heraud, W Zhang, JA Kralovec, D McNaughton, CJ Barrow. Quantitative determination of fatty acid compositions in micro-encapsulated fish-oil supplements using fourier transform infrared (FTIR) spectroscopy. Food Chem 2012;135:603-9.

11. Doolaanea AA, FM Ahmad, Fahmi Haruna. Quantification of Nigella sativa oil (NSO) from biodegradable PLGA nanoparticles using FTIR spectroscopy. Int J Pharm Pharm Sci 2014;6:228-32.

12. Doolaanea AA, NI Mansor, NH Mohd Nor, F Mohamed. Coencapsulation of Nigella sativa oil and plasmid DNA for enhanced gene therapy of Alzheimer's disease. J Microencapsul 2016;33:114-26.

13. Yang H, J Irudayaraj, MM Paradkar. Discriminant analysis of edible oils and fats by FTIR, FT-NIR and FT-Raman spectroscopy. Food Chem 2005;93:25-32.

14. Reddy KR, M Nagabhushanam. Process and parameters affecting drug release performance of prepared cross-linked alginate hydrogel beads for ezetimibe. Int J Pharm Pharm Sci 2017;9:254-62.

15. Pearce KN, JE Kinsella. Emulsifying properties of proteins: evaluation of a turbidimetric technique. J Agric Food Chem 1978;26:716-23.

16. Aoki T, EA Decker, DJ McClements. Influence of environmental stresses on stability of $\mathrm{O} / \mathrm{W}$ emulsions containing droplets stabilized by multilayered membranes produced by a layer-bylayer electrostatic deposition technique. Food Hydrocoll 2005;19:209-20.

17. Durante M, MS Lenucci, B Laddomada, G Mita, S Caretto. Effects of sodium alginate bead encapsulation on the storage stability of durum wheat (Triticum durum Desf.) bran oil extracted by supercritical CO2. J Agric Food Chem 2012;60:10689-95.

18. Reig FB, JG Adelantado, MM Moreno. FTIR quantitative analysis of calcium carbonate (calcite) and silica (quartz) mixtures using the constant ratio method. Application to geological samples. Talanta 2002;58:811-21.

19. Almeida PF, A Almeida. Cross-linked alginate-gelatine beads: a new matrix for controlled release of pindolol. J Controlled Release 2004;97:431-9.

20. Guillen M, N Cabo. Characterization of edible oils and lard by fourier transform infrared spectroscopy. Relationships between composition and frequency of concrete bands in the fingerprint region. J Am Oil Chem Soc 1997;74:1281-6.

21. Zeeb B, AH Saberi, J Weiss, DJ McClements. Retention and release of oil-in-water emulsions from filled hydrogel beads composed of calcium alginate: impact of emulsifier type and pH. Soft Matter 2015;11:2228-36. 